\title{
Minireview
}

\section{Progress in the analysis of membrane protein structure and function}

\author{
P.J.L. Werten ${ }^{\mathrm{a}}$, H.-W. Rémigy ${ }^{\mathrm{a}}$, B.L. de Groot ${ }^{\mathrm{b}}$, D. Fotiadis ${ }^{\mathrm{a}}$, A. Philippsen ${ }^{\mathrm{a}}$, H. Stahlberg ${ }^{\mathrm{a}}$, \\ H. Grubmüller ${ }^{\mathrm{b}}$, A. Engel ${ }^{\mathrm{a}, *}$ \\ ${ }^{a}$ M.E. Müller Institute for Microscopy, Biozentrum, University of Basel, Klingelbergstrasse 70, Basel CH-4056, Switzerland \\ ${ }^{\mathrm{b}}$ Theoretical Molecular Biophysics Group, Max-Planck-Institute for Biophysical Chemistry, Am Faßberg 11, 37077 Göttingen, Germany
}

Received 19 August 2002; accepted 20 August 2002

First published online 28 August 2002

Edited by Gunnar von Heijne

\begin{abstract}
Structural information on membrane proteins is sparse, yet they represent an important class of proteins that is encoded by about $30 \%$ of all genes. Progress has primarily been achieved with bacterial proteins, but efforts to solve the structure of eukaryotic membrane proteins are also increasing. Most of the structures currently available have been obtained by exploiting the power of X-ray crystallography. Recent results, however, have demonstrated the accuracy of electron crystallography and the imaging power of the atomic force microscope. These instruments allow membrane proteins to be studied while embedded in the bi-layer, and thus in a functional state. The low signal-to-noise ratio of cryo-electron microscopy is overcome by crystallizing membrane proteins in a two-dimensional proteinlipid membrane, allowing its atomic structure to be determined. In contrast, the high signal-to-noise ratio of atomic force microscopy allows individual protein surfaces to be imaged at subnanometer resolution, and their conformational states to be sampled. This review summarizes the steps in membrane protein structure determination and illuminates recent progress. (C) 2002 Published by Elsevier Science B.V. on behalf of the Federation of European Biochemical Societies.
\end{abstract}

Key words: Membrane protein expression;

Two-dimensional crystallization; Atomic force microscopy; Three-dimensional electron microscopy;

Molecular dynamics simulation

\section{Introduction}

Biological membranes fulfill vital functions as interfaces to the outside world, as interfaces between cells, and as boundaries of intracellular compartments. Thus, biological membranes are related to numerous diseases such as hyperinsulinemia, nephrogenic diabetes insipidus, congestive heart failure, liver cirrhosis, cystic fibrosis, hyper- and hypotension, lung edema, epilepsy, and cataract. About 30\% of the sequenced genes code for membrane proteins. However, only 30 unique structures of membrane proteins have been solved to atomic resolution, compared to 3000 unique crystal structures of soluble proteins, because it is difficult to produce three-dimensional (3D) crystals suitable for X-ray analyses from detergent-solubilized membrane proteins. Among the 67 membrane protein structures deposited in the protein

*Corresponding author. Fax: (41)-61-267 2109.

E-mail address: andreas.engel@unibas.ch (A. Engel). data base, 52 are of bacterial origin, suggesting that bacterial membrane proteins are more easily produced, purified and crystallized than those from plants or animals. The challenge now is to solve the structure of membrane proteins from higher organisms and to study their function, dynamics and interaction with ligands.

Progress in 3D crystallization of membrane proteins is distinct: the rate of new structures has recently increased [1-8], and $3 \mathrm{D}$ crystals of the first animal channel protein, bovine aquaporin-1 (AQP1), have been produced [9] and its structure has been solved to $2.2 \AA$ resolution [10]. The strength of this approach is the well-established technology that allows data to be collected and structures to be solved to high resolution with enormous efficiency. Nevertheless, this route to establish the atomic structure of a membrane protein is still risky as a result of the crystallization bottleneck. Solution nuclear magnetic resonance (NMR) is the other well-established method for determining atomic structures. It does not require $3 \mathrm{D}$ crystals and, additionally, allows the dynamics of a protein to be measured. Furthermore, progress towards assessing the structure of large complexes has been reported [11]. Difficulties with the stability of solubilized membrane proteins, however, can be a problem. A powerful alternative to determine the structure of a membrane protein is its reconstitution into two-dimensional (2D) membrane protein crystals in the presence of lipids [12]. This approach restores the native environment of membrane proteins as well as their biological activity. Cryo-electron microscopy (EM) allows the assessment of the 3D membrane protein structure at close to atomic resolution. The atomic force microscope (AFM) depicts biological membranes in aqueous solutions, and permits the movement of single polypeptide loops to be monitored [13]. In addition, this instrument allows the energetics of membrane protein interactions with their ligands to be assessed [14,15].

Here we review the steps in membrane protein structure determination. Expression and purification of membrane proteins must be optimized for all structure determination techniques. While 3D crystallization methods of membrane proteins have been reviewed recently [16], we summarize current methods of $2 \mathrm{D}$ crystallization. The examples that are presented here document the power of atomic force microscopy as well as EM. Finally, we concentrate on the recent progress resulting from the combination of microscopic techniques, structural bioinformatics, and molecular dynamics simulations to elucidate the structure and function of membrane proteins. 


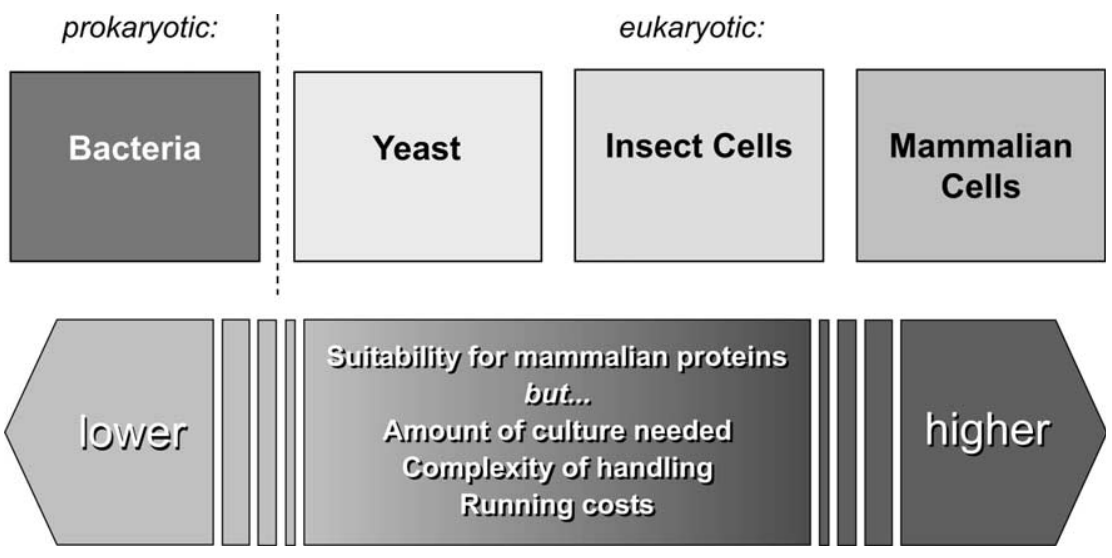

Fig. 1. Evaluation of the existing major expression systems.

\section{Protein expression, solubilization and purification}

Membrane proteins can often not be purified from natural sources in the amounts required for structural analyses. This is especially true for proteins that are normally expressed at very low levels or for proteins of human origin. The use of efficient heterologous expression systems as a source of abundant protein is therefore essential. Currently, four major expression systems can be distinguished: those using bacteria, yeast, insect cells, or mammalian cells for expression. It should be obvious that any recombinant protein can be best expressed in the expression system that most closely resembles the natural environment of the protein. For mammalian proteins, therefore, a mammalian expression system is likely to give the best results in terms of structure and functionality of the protein. Indeed, mammalian membrane proteins often require specific lipid environments, the presence of certain chaperones, and specific post-translational modifications. It is well known that the use of prokaryotic or lower eukaryotic expression systems for mammalian proteins can lead to misfolding or loss of functionality of the protein expressed [17]. From a production point of view, however, higher eukaryotic expression systems are much more expensive, more complex in their handling and often yield less protein per liter of cell culture than their simpler counterparts (see Fig. 1). It makes sense, therefore, to check expression of a new recombinant protein in all systems available at least until the stage where functionality and yield can be assessed, and only then decide which system is best suited for large-scale production. For this purpose, a well-designed and flexible cloning procedure, allowing easy cloning of the cDNA of interest into the various systems, is a requirement.

Recently, a very efficient system for the expression of proteins in mammalian cells was developed, not requiring the often difficult and time-consuming process of making stable cell lines [18]. With this system, cell lines of various eukaryotic origins can be infected by the Semliki Forest Virus carrying the gene of interest under the control of its viral promoter. For safety reasons, the virus has been made replication deficient by splitting the RNA replication genes and structural genes into two different vectors. As a further safety mechanism, the virus has to be activated by chymotrypsin. In this way, a transient but highly effective protein production can take place in a cell line of choice.

After protein production, an intensive screening of deter- gents is used to identify the detergent that (1) solubilizes the protein efficiently, (2) allows for purification of the protein (e.g. via Ni-affinity chromatography), (3) does not disturb the structure and function of the protein, and (4) allows reconstitution into $2 \mathrm{D}$ crystals (see below). In addition, some form of prior stripping of the membranes may be required to remove membrane-associated proteins [19-21], thereby making the membranes more accessible to detergents. Once solubilized, the protein can either be purified via classical chromatographic methods or, more conveniently, via affinity purification using tags present in the protein (e.g. His-tag, FLAG-tag, HA-tag, Streptavidin-tag, etc.). The latter method is quick and often yields highly pure protein in a single purification step, but tags can change the properties of the protein and should ideally be cleaved off after purification. Purified and solubilized protein can conveniently be studied by EM of negatively stained samples. The homogeneity of such preparations is readily checked and the stability of the protein in the detergent used can thus be evaluated. Long-term stability may also be checked in this manner.

\section{3. $2 \mathrm{D}$ crystallization}

Because biomolecules are beam sensitive and produce a low image contrast, extensive image averaging is required to retrieve high-resolution structural information by cryo-EM. Averaging can most efficiently be carried out when the molecules are packed in a $2 \mathrm{D}$ crystal. With membrane proteins $2 \mathrm{D}$ crystallization also offers the advantage of restoring their native environment: by exploiting their amphiphilic character, membrane proteins can be packed into a $2 \mathrm{D}$ crystal in the presence of lipids. Such 2D crystals therefore warrant full activity of the protein and, importantly, also preserve the native conformation of hydrophilic loops that are typically required for interaction with ligands. In $3 \mathrm{D}$ crystals, these are often immobilized in crystal contacts. On the other hand, 3D crystals are larger, often better ordered and house $10^{6}-10^{8}$ times more unit cells than $2 \mathrm{D}$ crystals, explaining why it is easier to obtain high resolution by X-ray crystallography.

After isolation in detergents, solubilized membrane proteins are reconstituted into a lipid bi-layer at a lipid-to-protein ratio of close to one (weight-to-weight). The assembly of lipids and proteins is driven by detergent removal, which is the main approach used for 2D crystallization (Fig. 2). While this principle of $2 \mathrm{D}$ crystallization is rather general, the recipe working 


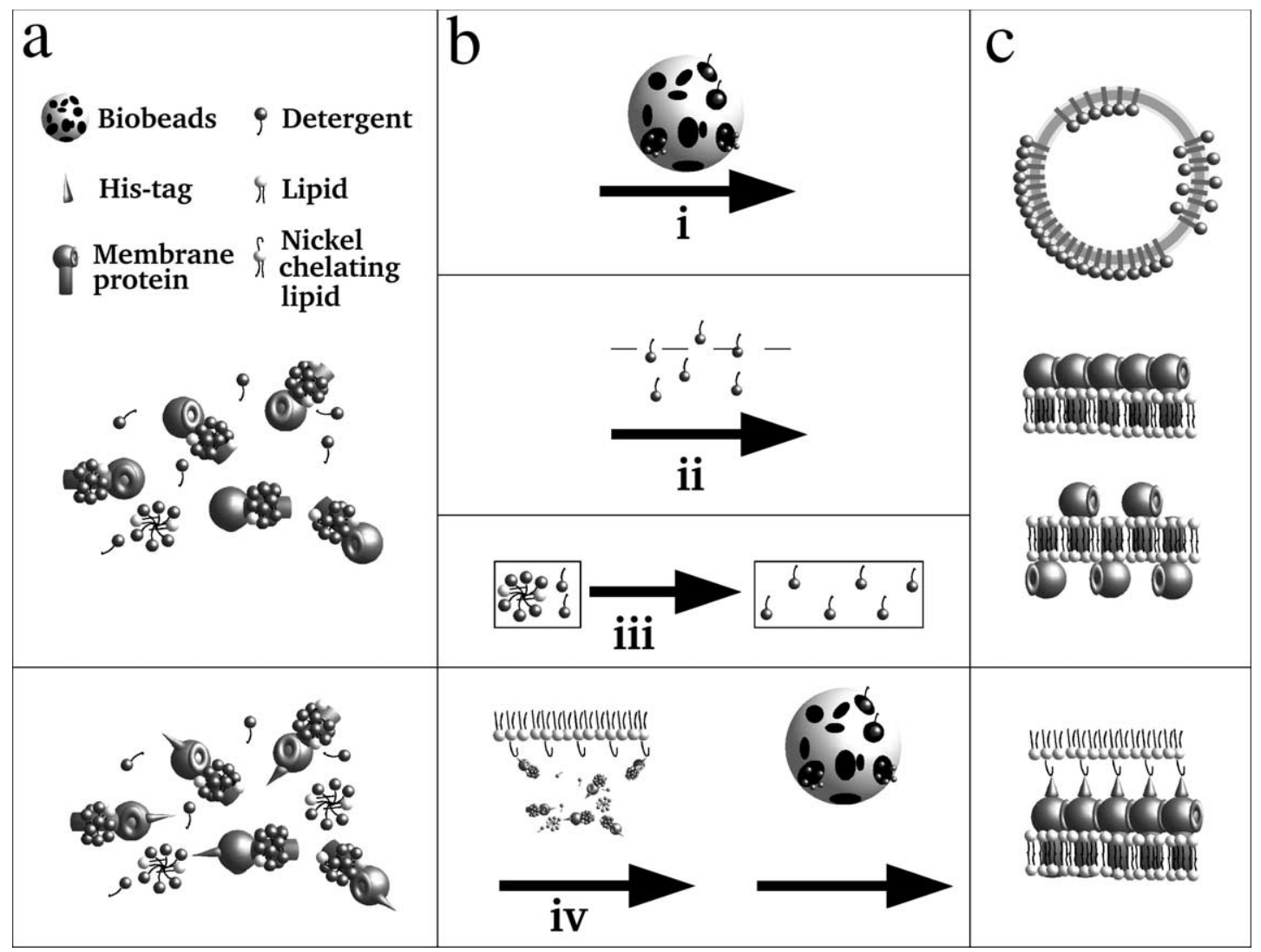

Fig. 2. 2D crystallization by the detergent removal principle. A: Starting conditions: detergent-solubilized proteins are mixed with lipid-detergent micelles. B: Detergent removal using (i) Biobeads [23], (ii) dialysis [24], (iii) dilution [22], and (iv) Biobeads combined with a monolayer at the air-water interface, which binds His-tagged proteins [25]. C: The resulting 2D crystals can exist as a vesicular or a sheet-like assembly, or they are trapped at the air-water interface.

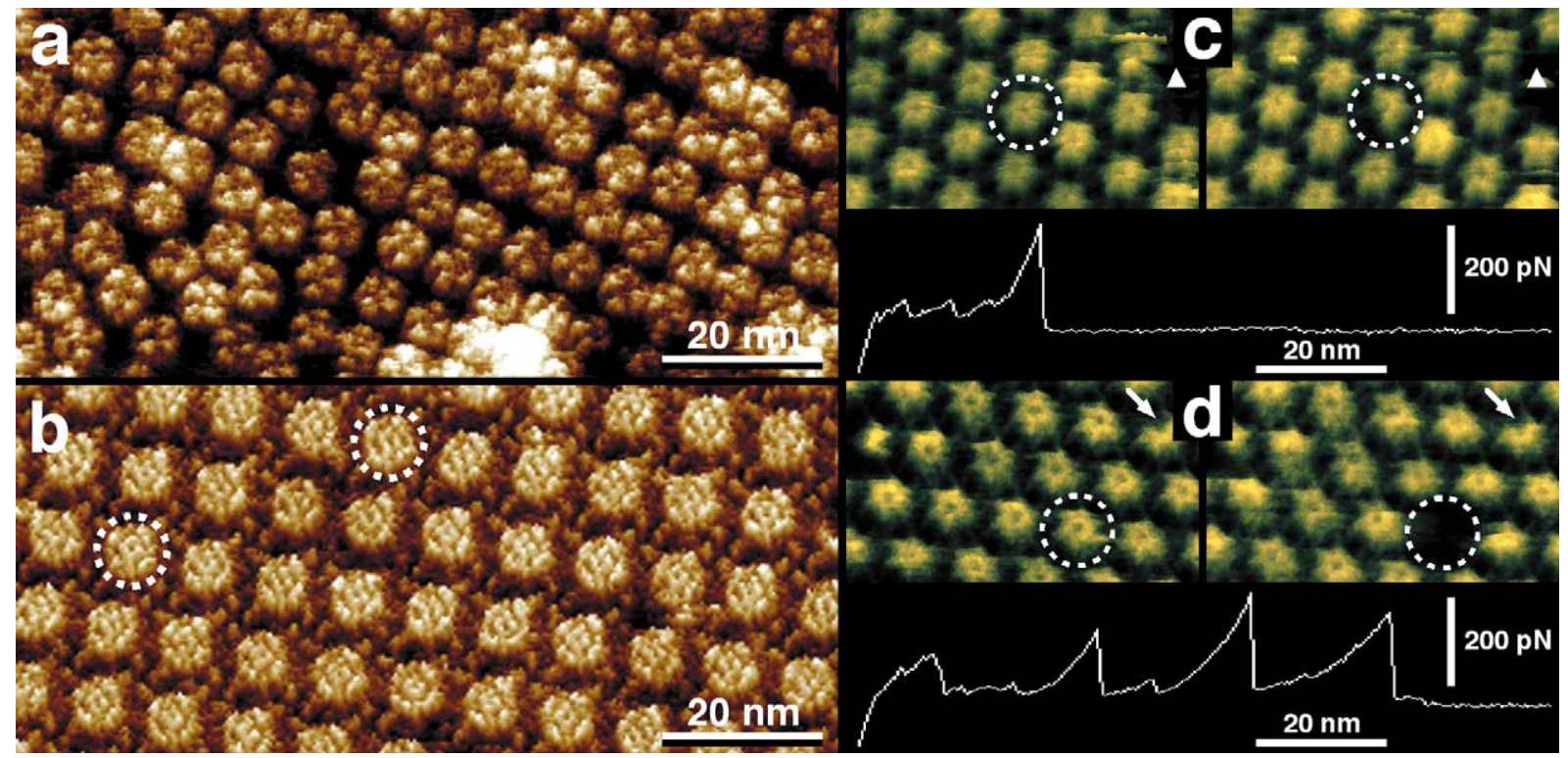

Fig. 3. Atomic force microscopy of biomolecules imaged in aqueous solution. A: Surface topograph of a densely packed vesicle containing tetramers of the major intrinsic protein from lens fiber cells [29]. B: Topograph of a 2D crystal of AqpZ from Escherichia coli [13]. Single tetramers exposing the periplasmic side are marked by broken circles. C: Topographs of the surface layer of Corynebacterium glutamicum before (left panel) and after (right panel) removal of two protomers [32]. The bottom panel displays the force-distance curve of the induced unfolding giving rise to the surface change (broken circles) observed on the panels above. D: Same as for C, except that here all six protomers were removed. The triangles and the arrows in panels C and D mark defects in the layer that facilitate orientation. 
for one membrane protein can rarely be applied to another. Purification procedure, $\mathrm{pH}$, ionic strength, detergents and the specific, protein-bound lipids significantly affect protein stability. For $2 \mathrm{D}$ crystallization, the protein concentration, choice of lipids and detergent as well as the reconstitution kinetics all are crucial factors whose influence is not well predictable [2224]. Therefore, these factors must be screened to determine which conditions are suitable for the protein and will most likely result in 2D crystals. Results are continuously checked by EM of negatively stained samples.

Four main techniques are used for 2D crystallization (Fig. 2): (i) the Biobead method (detergent but not lipid is trapped in nanoscale cavities of polystyrene beads [23]); (ii) the dialysis method [24]; (iii) the dilution method (the lipid-detergent-protein mixture is diluted to bring the detergent below the critical-micellar concentration (CMC) [22]); and (iv) the monolayer method (the ternary complex is bound to a functionalized lipid monolayer at the air-water interface and Biobeads remove the detergent [25]). Each technique has its own advantages: the use of Biobeads permits fast and complete removal of low- as well as high-CMC detergents. Dialysis is the most successful crystallization method and has produced the best $2 \mathrm{D}$ crystals, although it works efficiently only with high-CMC detergents. The main advantage of dilution is to have a complete control of reconstitution kinetics. Thus, the process can be slowed down when a favorable equilibrium between proteins, detergents and lipids for $2 \mathrm{D}$ crystallization is reached. Because the final protein concentration is an issue in crystallization in general, dilution has its limitation. A nickel-chelating lipid monolayer forces His-tagged membrane proteins to be in close contact and to form a monolayer as well, facilitating the $2 \mathrm{D}$ crystallization of unidirectionally oriented proteins. This concentrating effect permits the design of reconstitution experiment with small amounts of proteins.

\section{Atomic force microscopy}

The atomic force microscope (AFM) [26] measures the surface topography by raster scanning the sample laterally below a sharp stylus that is attached to a flexible cantilever. A servo system controls the scanner, displacing the sample also vertically to keep the cantilever deflection constant. An optical detector resolves deflections of $0.1 \mathrm{~nm}$, which corresponds to a force difference of typically $10-50 \mathrm{pN}$. Thus state-of-the-art instruments can contour sample topographies in aqueous solutions at forces in this range. By electrostatically balancing the forces between tip and sample, topographs are recorded without sample deformation, yielding a lateral resolution that is usually better than $1 \mathrm{~nm}$ and a vertical resolution of typically $0.1 \mathrm{~nm}$ [27]. In combination with high-resolution imaging, the AFM offers the unique possibility of manipulating biologically active macromolecules under physiological conditions [28]. A further feature of the AFM is the outstanding signal-to-noise ratio provided in the topographs. As illustrated by Fig. 3A, single membrane protein complexes (left half) and densely packed, non-crystalline arrays thereof (right half) can be imaged at sub-nanometer resolution [29]. Such imaging capacity makes the AFM the instrument of choice to assess the structure and oligomeric state of single membrane proteins within native membranes [30]. This imaging capacity can also be exploited to observe single biomolecules at work [31] and to sample the conformational space and energy landscape of a membrane protein surface [13]. Fig. 3B shows the AFM topography of a $2 \mathrm{D}$ crystal of aquaporin-Z (AqpZ). The conformational variability on the periplasmic surface of single AqpZ tetramers (broken circles) can clearly be seen. The sensitivity of the cantilever deflection detector has made single molecule force measurements with the AFM possible $[14,15]$. Force-distance curves are recorded by vertically displacing the tip towards the sample until a single molecule is attached by contact-adhesion. Forces developing during subsequent tip-retraction reflect the induced unfolding pathway of the protein. Acquisition of high-resolution images before and after 'unzipping' a biomolecule allows the damage produced to be directly visualized (Fig. 3C and D) [32,33].

\section{Electron microscopy}

Transmission EM has progressed steadily, and modern instruments equipped with a field-emission gun (FEG) are now available that efficiently transfer the atomic scale structural information from the sample to the image, which represents a projection of the $3 \mathrm{D}$ potential distribution of the object. Meaningful information, however, can only be extracted when the sample is structurally preserved in spite of the vacuum within the electron optical system. Suitable methods produce biomolecules embedded in a thin vitrified water layer $[34,35]$, or embedded in a layer of partially dried and frozen sugar solution [36]. A combination of staining the sample with a heavy metal salt to enhance the contrast, and freezing the sample to preserve the high-resolution structure is found in the cryo-negative stain sample preparation method [37]. Since all these samples are highly sensitive to the electron beam, images are recorded at low electron doses. High-resolution data of proteins are recorded at doses below 5 electrons $/ \AA^{2}$, with the sample kept at liquid nitrogen temperature $(77 \mathrm{~K})$ [38], or at doses below 20 electrons $/ \AA^{2}$, when the sample is kept at liquid helium temperature $(4.3 \mathrm{~K})$ [39]. Such recording doses produce inherently noisy images, whose information must be extracted by image averaging.

Since images (rather than diffraction patterns) are recorded, thousands of single particle projections can be acquired, classified, aligned, and averaged $[40,41]$. In this way the complete information for a full 3D reconstruction of the potential map is collected without producing any crystals of the particles. Single particle reconstruction has mainly been used for large soluble complexes such as the ribosome $[42,43]$, but also for some solubilized membrane proteins [44]. Due to the limited size of the individual particles, precise alignment of the projections requires that the highest possible resolution and contrast are available. Therefore, the use of a highly coherent electron beam produced by a FEG is mandatory $[39,41]$. The achievable resolution of single particle structure determination is now getting better than $10 \AA$.

Images of $2 \mathrm{D}$ crystals of membrane proteins, however, allow the image signal to be extracted by crystallographic methods. Using this approach, the atomic structure of bacteriorhodopsin (bR) was first determined [45], and subsequently refined [46-48] using prototype FEG microscopes while keeping the sample at a few Kelvin. Two other atomic structures of membrane proteins were subsequently solved using similar instruments: the light-harvesting complex of plants [49] and human aquaporin-1 (AQP1) [50]. Recent progress in 2D crystallization has delivered crystals of several transporters (NhaA 

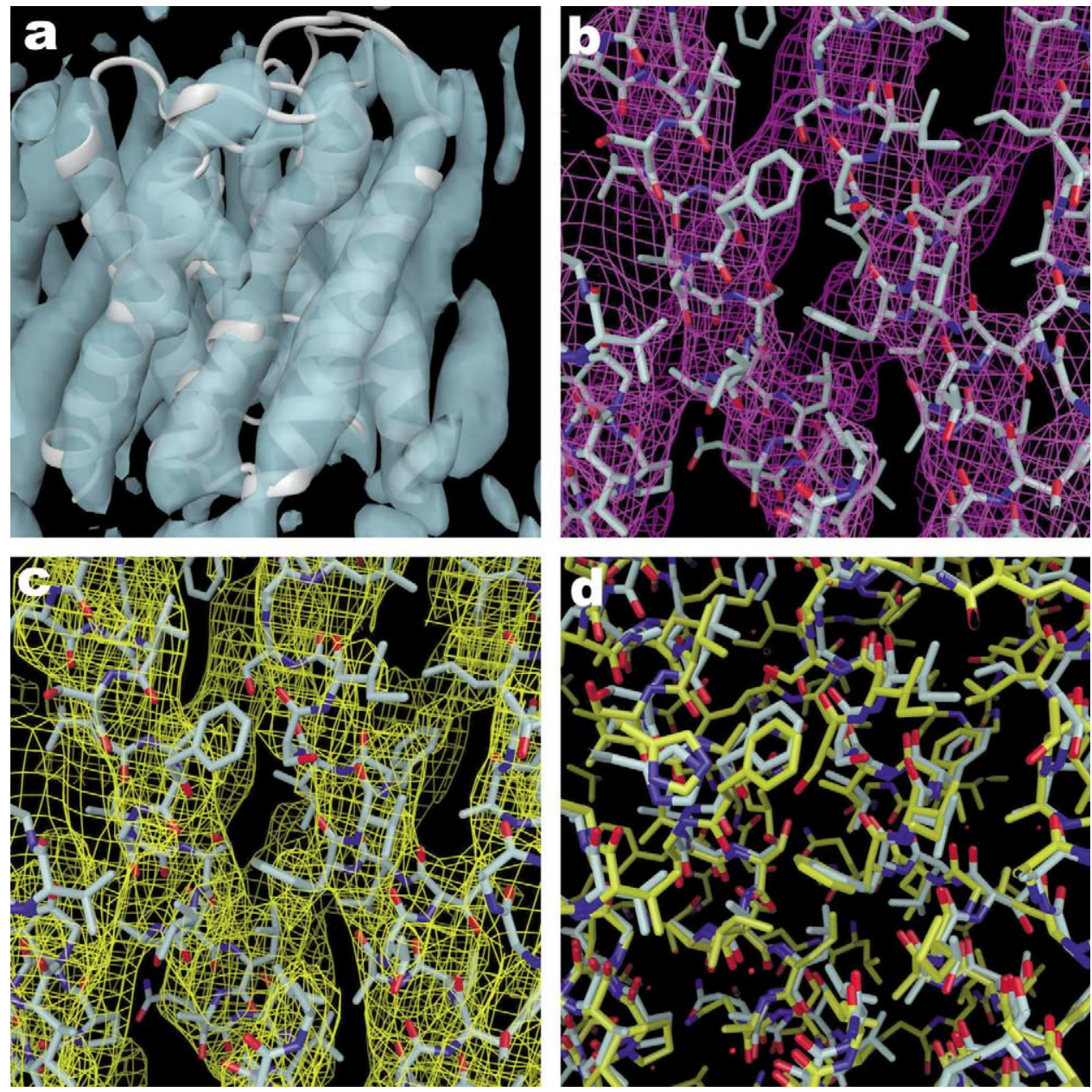

Fig. 4. Progress in the elucidation of human AQP1. A: At $6 \AA$ resolution the secondary structure becomes visible [71]. B: At 4.5 $\AA$ resolution the details suffice to place the peptide backbone [72], side chains usually not being discernible in the map. C: At $3.8 \AA$ resolution side chains become distinct. An atomic model can be built by hand [50] and refined using structural bioinformatics [60]. D: The refined structure (in gray) is compared with the $2.2 \AA$ structure (in yellow) obtained by X-ray crystallography [10]. The figure was generated with DINO (www.dino3d. org).

[51], OxlT [52], and SecYEG [53]), of the bacterial rotor of ATPase [54,55], and of a ClC-type chloride channel [56], which allowed structure determination to a sufficient resolution (approximately $6 \AA$ ) for visualizing the secondary structure.

Interpreting a cryo-EM potential map in terms of atomic protein structure typically still is a challenging task, even when relatively high-resolution data of better than $4 \AA$ are available. The first step is to determine the macromolecular fold and to trace the backbone through the map. In the case of membrane proteins, this can be a non-trivial task: although secondary structure elements like helices and strands may be clearly distinguishable, the map is frequently poorly defined in the loop regions. Nevertheless, a number of approaches have been applied to tackle this problem. First, the backbone trace (and thereby the 3D fold) can be derived by visual inspection. In such cases, the presence of a limited number of bulky groups at particular sites is a prerequisite for the successful identification of their location in the experimental map. This approach was used for $\mathrm{bR}$, the first membrane protein structure to be solved by EM $[45,46]$. Further, structural clues can be obtained from bioinformatics methods. Extensive sequence alignments and the analysis of correlated mutations led to valuable insights in the case of AQP1 [57]. Finally, in the case of $\alpha$-helical proteins, automated procedures have been developed to determine both the location and direction of individual helices in the map $[58,59]$. When used in conjunction with constraints from the sequence (e.g. the maximal length of a loop), macromolecular folds can be unambiguously derived this way.

Once the fold has been determined, an initial model of the backbone structure can readily be generated. Depending on the resolution, what follows are many rounds of manual model (re)building and refinement. The structures of bR [45-48] and AQP1 [50,60,61] (Fig. 4) have been solved by EM in this way and were later confirmed by X-ray crystallography $[7,10]$. These successes demonstrate that even at a resolution of around $3.5 \AA$, a cryo-EM map contains sufficient structural information to uniquely define the atomic structure. The practical challenge is to find this optimal structure in the highdimensional search space. Since an exhaustive search is not feasible, there is no straightforward general method to arrive at the correct (or best possible) solution at this level of resolution. The available tools are for a substantial part the same as used in X-ray crystallography but, if used inappropriately, the usually lower resolution may lead to inaccurate interpre- 


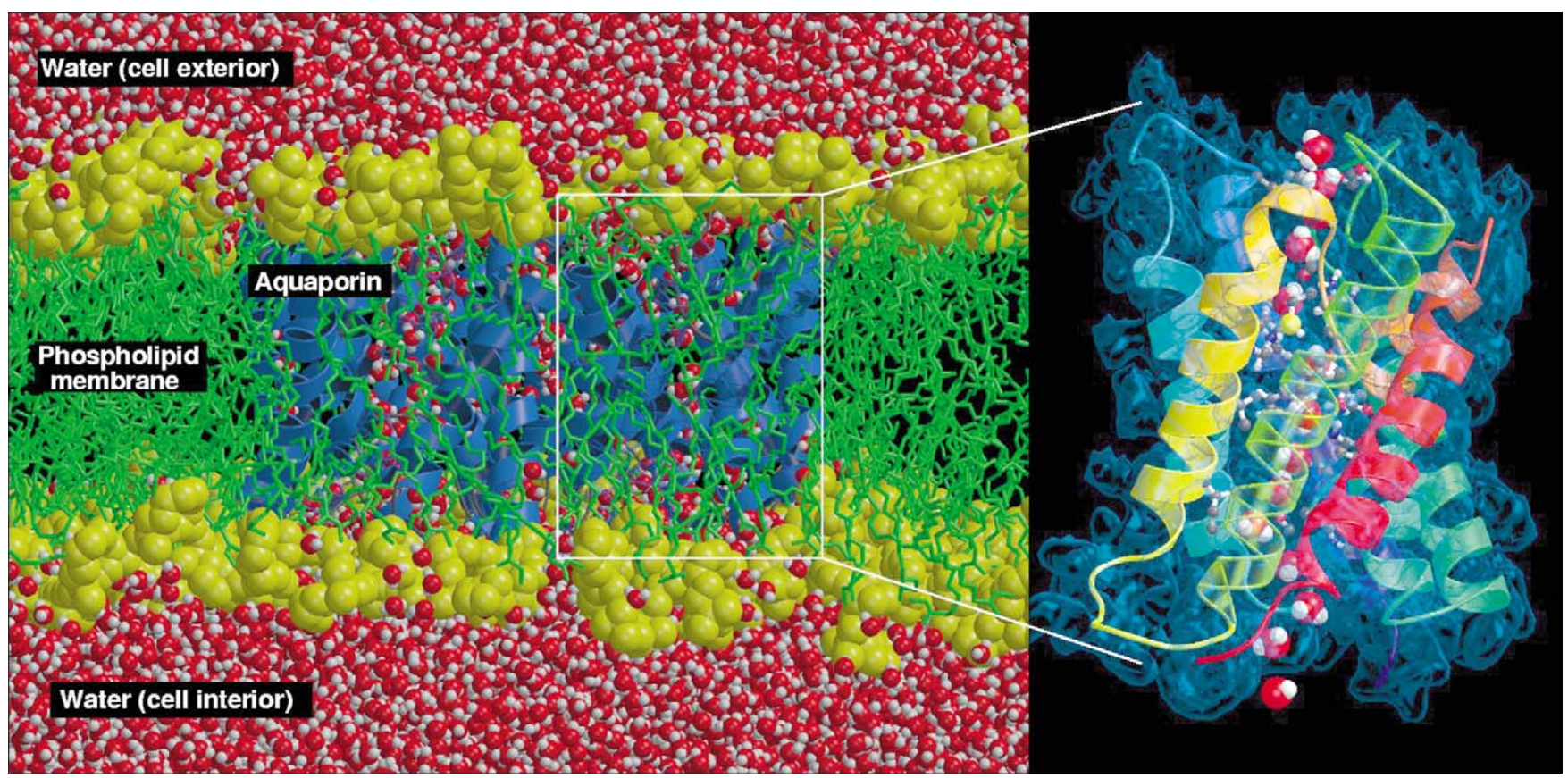

Fig. 5. Multi-nanosecond molecular dynamics simulation of water permeation through AQP1 [64]. Left: The simulation system of the AQP1 tetramer (blue) embedded in a lipid bi-layer (yellow/green) surrounded by water (red/white) consisted of approximately 100000 atoms. Right: Pathway of a water molecule for one of the spontaneous permeation events observed in the simulations. All water molecules were found to be strongly oriented in the channel interior, with their dipoles rotating by about $180^{\circ}$ during permeation.

tations of the data, slow convergence of the refinement, or poor model quality. In contrast to X-ray crystallography, experimental phase information is available in the case of EM. Unfortunately, this advantage is not as of yet fully exploited. Currently, only a trained eye can incorporate all the available structural information into a model. Inevitably however, all manual intervention potentially leads to model bias. Therefore, the development of novel automated techniques specifically targeted at model building and refinement based on EM data is mandatory.

\section{Function}

While crystallographic methods provide atomic resolution, they reveal only the static part of a structure. Although distinct conformations can sometimes be trapped and resolved with these techniques, biomolecules in Brownian motion typically adopt many other conformations that remain invisible. NMR spectroscopy and AFM can provide insight into such processes. While the NMR method gives atomic scale information of entire ensembles, the AFM allows single molecules to be monitored over time, albeit at a lower resolution. Single molecule dynamics, however, can be simulated computationally, allowing the dynamics of biomolecular processes to be monitored atom by atom at ultra-high time resolution. Current molecular dynamics simulations can be carried out on systems of more than $10^{5}$ atoms over tens of nanoseconds, giving a detailed insight into the function of proteins.

A recent example is the simulation of water flow through specific membrane pores. To filter 180 liters of blood per day in the kidneys, water must permeate through human AQP1 at a rate of 3 billion water molecules per second [62,63]. Such amazing rates are typical for all aquaporins and raise the question as to how these highly specific channels allow water to pass unhindered but prevent the flow of protons. Molecular dynamics simulations of systems comprising either an AQP1 tetramer or a glycerol permeation facilitator $(\mathrm{GlpF})$ tetramer, which are embedded in a lipid bi-layer and surrounded by water molecules, provided a detailed time-resolved picture of the water permeation and ion-blockage mechanisms [64,65] (Fig. 5). The simulations showed spontaneous, bi-directional, full water permeation events (Fig. 5, right), with rates in close agreement to the experimentally determined rates [64]. For the related glycerol facilitator GlpF, spontaneous partial glycerol permeation events, involving all three glycerol molecules per channel as observed by X-ray crystallography [3], were also observed in simulations [66]. Subsequent steered molecular dynamics simulations allowed the computation of a free energy profile for glycerol motion along the channel [67].

In the simulations of both aquaglyceroporins, water molecules were found to be strongly oriented in the channel interior, through a fine-tuned alignment of their dipoles with the electric field exerted by the protein, causing water molecules to rotate by $180^{\circ}$ upon passage $[60,65]$ (Fig. 5, right). Simulations of the glycerol-free GlpF structure showed that the electrostatic interactions between water on the one hand, and the NPA motifs and the dipoles of helices HB and HE on the other hand, are the main cause for the observed water rotation [65]. Hydrogen bond competition between water molecules and the few polar groups in the pore was found to dominate the energetics of the permeation process [60]. Two major interaction sites for water molecules were identified inside the channel, the NPA and the aromatic/arginine constriction regions, which were found to weaken the hydrogen bond interactions along the water file in the channel. As contiguous hydrogen bonded water chains are known to be efficient proton conductors, it was suggested that these regions are the main proton filters $[60,65]$. From inspection of the charges 
along the channel, electrostatic barriers likely also contribute significantly to the proton filter, which needs to be studied in more detail [68].

\section{Conclusions and perspectives}

Within a decade, membrane protein structure analyses have progressed tremendously. A growing number of membrane proteins have been solved to atomic resolution mainly by $\mathrm{X}$-ray crystallography, which is now being successfully used by many laboratories. In addition, significant and steady progress has been made in atomic force microscopy and electron crystallography, although these techniques are being developed by only a small number of groups. These microscopic techniques cannot compete with X-ray or NMR techniques in terms of resolution. They do, however, deliver information that can be combined with structural bioinformatics and molecular dynamics simulations to establish a detailed understanding of membrane protein structure and function.

The first generation of commercial AFMs has produced a number of spectacular results that indicate the breadth of future applications. These will include high-resolution imaging of native biological membranes, the determination of membrane protein topology by single molecule force measurements, and the direct, time-resolved monitoring of functionrelated conformational changes. Such measurements will be enhanced by the next generation of scanning probe microscopes that is currently being developed by physicists [69].

High-resolution transmission electron microscopes exploiting highly coherent electron beams are now available that maintain low sample temperatures, warrant negligible drift, and that transfer the structural information to $2 \AA$ resolution. In spite of low sample temperatures that reduce beam damage, the fundamental bottle neck to overcome remains the beam sensitivity of biological matter. Single particle methods circumvent difficulties related to the crystallization of membrane proteins. However, to achieve close to atomic resolution by this approach is a truly ambitious endeavor [41]. In contrast, 2D crystals have been successfully used to reach atomic resolution. This approach is of particular interest for membrane proteins, because the native environment can be restored, as 2D crystals are reconstituted in the presence of lipids. As demonstrated with AQP1, 2D crystals can be utilized to assess the function of a membrane channel quantitatively, since the number of participating molecules is precisely known [70]. The first atomic model of AQP1 has been established by electron crystallography [50]. Refinement of this structure using structural bioinformatics has led to an atomic model [60], which allowed application of molecular dynamics simulations to study the mechanism of water transport [64]. With the high-resolution structure of AQP1 determined by X-ray analyses now available [10], the quality of the lower resolution structure from electron crystallography has been assessed and found to agree, even in significant details [68]. This recent comparison stimulates further efforts on electron crystallography, with the goal of improving both data processing technology as well as $2 \mathrm{D}$ crystallogenesis.

Improving instrumentation, sample preparation methods, and data retrieval and processing are difficult tasks, but progress is predictable and steady. In contrast, crystallization of membrane proteins remains an empirical discipline that is full of unexpected adversities. The stability and purity of protein preparations depend on many elusive factors that are closely related to expression and purification methods. Therefore, the ultimate bottleneck of atomic scale structural and functional analyses of membrane proteins will be their recombinant expression and subsequent crystallization.

Acknowledgements: This work was supported by the Swiss National Foundation, the M.E. Müller-Foundation of Switzerland, the European Union-Quality of Life and Management of Living Resources Project (Grants QLRT-2000-00778 and QLRT-2000-00504 to H.G. and A.E.), the NCCR 'Nanoscale Science', the NCCR 'Structural Biology', and the Human Frontier Science Program (Grant RG0021/2000-M103 to A.E.).

\section{References}

[1] Chang, G., Spencer, R.H., Lee, A.T., Barclay, M.T. and Rees, D.C. (1998) Science 282, 2220-2226.

[2] Doyle, D.A., Cabral, J.M., Pfuetzner, R.A., Kuo, A., Gulbis, J.M., Cohen, S.L., Chait, B.T. and MacKinnon, R. (1998) Science $280,69-77$.

[3] Fu, D., Libson, A., Miercke, L.J., Weitzman, C., Nollert, P., Krucinski, J. and Stroud, R.M. (2000) Science 290, 481-486.

[4] Hunte, C., Koepke, J., Lange, C., Rossmanith, T. and Michel, H. (2000) Struct. Fold Des. 8, 669-684.

[5] Lancaster, C.R. (2001) FEBS Lett. 504, 133-141.

[6] Locher, K.P., Lee, A.T. and Rees, D.C. (2002) Science 296, 1091-1098

[7] Luecke, H., Schobert, B., Richter, H.T., Cartailler, J.P. and Lanyi, J.K. (1999) J. Mol. Biol. 291, 899-911.

[8] Pebay-Peyroula, E., Rummel, G., Rosenbusch, J.P. and Landau, E.M. (1997) Science 277, 1676-1681.

[9] Sui, H., Walian, P.J., Tang, G., Oh, A. and Jap, B.K. (2000) Acta Crystallogr. Sec. D 56, 1198-1200.

[10] Sui, H., Han, B.G., Lee, J.K., Walian, P. and Jap, B.K. (2001) Nature 414, 872-878.

[11] Fiaux, J., Bertelsen, E.B., Horwich, A.L. and Wüthrich, K. (2002) Nature 418, 207-211.

[12] Stahlberg, H., Fotiadis, D., Scheuring, S., Remigy, H., Braun, T., Mitsuoka, K., Fujiyoshi, Y. and Engel, A. (2001) FEBS Lett. $504,166-172$

[13] Scheuring, S., Müller, D.J., Stahlberg, H., Engel, H.A. and Engel, A. (2002) Eur. Biophys. J. 31, 172-178.

[14] Clausen-Schaumann, H., Seitz, M., Krautbauer, R. and Gaub, H.E. (2000) Curr. Opin. Chem. Biol. 4, 524-530.

[15] Fisher, T.E., Marszalek, P.E. and Fernandez, J.M. (2000) Nat. Struct. Biol. 7, 719-724.

[16] Rosenbusch, J.P. (2001) J. Struct. Biol. 136, 144-157.

[17] Tate, C.G. (2001) FEBS Lett. 504, 94-98

[18] Lundstrom, K. (2000) Intervirology 43, 247-257.

[19] Kistler, J., Goldie, K., Donaldson, P. and Engel, A. (1994) J. Cell Biol. 126, 1047-1058.

[20] Werten, P.J.L., Hasler, L., Koenderink, J.B., Klaassen, C.H.W., de Grip, W.J., Engel, A. and Deen, P.M.T. (2001) FEBS Lett. 504, 200-205.

[21] Fotiadis, D., Jeno, P., Mini, T., Wirtz, S., Müller, S.A., Fraysse, L., Kjellbom, P. and Engel, A. (2001) J. Biol. Chem. 276, 17071714.

[22] Dolder, M., Engel, A. and Zulauf, M. (1996) FEBS Lett. 382, 203-208.

[23] Rigaud, J.-L., Pitard, B. and Levy, D. (1995) Biochim. Biophys. Acta 1231, 223-246.

[24] Hasler, L., Heymann, J.B., Engel, A., Kistler, J. and Walz, T. (1998) J. Struct. Biol. 121, 162-171.

[25] Levy, D., Chami, M. and Rigaud, J.L. (2001) FEBS Lett. 504, $187-193$.

[26] Binnig, G., Quate, C.F. and Gerber, C. (1986) Phys. Rev. Lett. 56, 930-933.

[27] Müller, D.J., Fotiadis, D., Scheuring, S., Müller, S.A. and Engel, A. (1999) Biophys. J. 76, 1101-1111.

[28] Fotiadis, D., Scheuring, S., Müller, S.A., Engel, A. and Müller, D.J. (2002) Micron 33, 385-397.

[29] Fotiadis, D., Hasler, L., Müller, D.J., Stahlberg, H., Kistler, J. and Engel, A. (2000) J. Mol. Biol. 300, 779-789. 
[30] Scheuring, S., Reiss-Husson, F., Engel, A., Rigaud, J.L. and Ranck, J.L. (2001) EMBO J. 20, 3029-3035.

[31] Engel, A. and Müller, D.J. (2000) Nat. Struct. Biol. 7, 715-718.

[32] Scheuring, S., Stahlberg, H., Chami, M., Houssin, C., Rigaud, J.L. and Engel, A. (2002) Mol. Microbiol. 44, 675-684.

[33] Oesterhelt, F., Oesterhelt, D., Pfeiffer, M., Engel, A., Gaub, H.E. and Müller, D.J. (2000) Science 288, 143-146.

[34] Adrian, M., Dubochet, J., Lepault, J. and McDowall, A.W. (1984) Nature 308, 32-36.

[35] Dubochet, J., Adrian, M., Chang, J.-J., Homo, J.-C., Lepault, J., McDowall, A.W. and Schultz, P. (1988) Q. Rev. Biophys. 21, 129-228.

[36] Henderson, R. and Unwin, P.N. (1975) Nature 257, 28-32.

[37] Adrian, M., Dubochet, J., Fuller, S.D. and Harris, J.R. (1998) Micron 29, 145-160.

[38] Conway, J.F., Trus, B.L., Booy, F.P., Newcomb, W.W., Brown, J.C. and Steven, A.C. (1993) J. Struct. Biol. 111, 222-233.

[39] Fujiyoshi, Y. (1998) Adv. Biophys. 35, 25-80.

[40] Frank, J. (2002) Annu. Rev. Biophys. Biomol. Struct. 31, 303319.

[41] van Heel, M. et al. (2000) Q. Rev. Biophys. 33, 307-369.

[42] Frank, J., Penczek, P., Agrawal, R.K., Grassucci, R.A. and Heagle, A.B. (2000) Methods Enzymol. 317, 276-291.

[43] van Heel, M. (2000) Curr. Opin. Struct. Biol. 10, 259-264.

[44] Grigorieff, N. (1998) J. Mol. Biol. 277, 1033-1046.

[45] Henderson, R., Baldwin, J.M., Ceska, T.A., Zemlin, F., Beckmann, E. and Downing, K.H. (1990) J. Mol. Biol. 213, 899-929.

[46] Grigorieff, N., Ceska, T.A., Downing, K.H., Baldwin, J.M. and Henderson, R. (1996) J. Mol. Biol. 259, 393-421.

[47] Kimura, Y. et al. (1997) Nature 389, 206-211.

[48] Mitsuoka, K., Hirai, T., Murata, K., Miyazawa, A., Kidera, A., Kimura, Y. and Fujiyoshi, Y. (1999) J. Mol. Biol. 286, 861-882.

[49] Kühlbrandt, W., Wang, D.N. and Fujiyoshi, Y. (1994) Nature 367, 614-621.

[50] Murata, K., Mitsuoka, K., Hirai, T., Walz, T., Agre, P., Heymann, J.B., Engel, A. and Fujiyoshi, Y. (2000) Nature 407, 599605.

[51] Williams, K.A. (2000) Nature 403, 112-115.

[52] Hirai, T., Heymann, J.A., Shi, D., Sarker, R., Maloney, P.C. and Subramaniam, S. (2002) Nat. Struct. Biol. 9, 597-600.

[53] Breyton, C., Haase, W., Rapoport, T.A., Kühlbrandt, W. and Collinson, I. (2002) Nature 418, 662-665.
[54] Stahlberg, H., Müller, D.J., Suda, K., Fotiadis, D., Engel, A., Meier, T., Matthey, U. and Dimroth, P. (2001) EMBO Rep. 2, 229-233.

[55] Vonck, J., von Nidda, T.K., Meier, T., Matthey, U., Mills, D.J., Kühlbrandt, W. and Dimroth, P. (2002) J. Mol. Biol. 321, 307316.

[56] Mindell, J.A., Maduke, M., Miller, C. and Grigorieff, N. (2001) Nature 409, 219-223.

[57] Heymann, J.B. and Engel, A. (2000) J. Mol. Biol. 295, 10391053.

[58] de Groot, B.L., Heymann, J.B., Engel, A., Mitsuoka, K., Fujiyoshi, Y. and Grubmüller, H. (2000) J. Mol. Biol. 300, 987-994.

[59] Jiang, W., Baker, M.L., Ludtke, S.J. and Chiu, W. (2001) J. Mol. Biol. 308, 1033-1044.

[60] de Groot, B.L., Engel, A. and Grubmüller, H. (2001) FEBS Lett. 504, 206-211.

[61] Ren, G., Reddy, V.S., Cheng, A., Melnyk, P. and Mitra, A.K. (2001) Proc. Natl. Acad. Sci. USA 98, 1398-1403.

[62] van Hoek, A.N. and Verkman, A.S. (1992) J. Biol. Chem. 267, 18267-18269.

[63] Zeidel, M.L., Ambudkar, S.V., Smith, B.L. and Agre, P. (1992) Biochemistry 31, 7436-7440.

[64] de Groot, B.L. and Grubmüller, H. (2001) Science 294, 2353 2357.

[65] Tajkhorshid, E., Nollert, P., Jensen, M.Ø., Miercke, L.J., O'Connell, J., Stroud, R.M. and Schulten, K. (2002) Science 296, 525530.

[66] Jensen, M.Ø., Tajkhorshid, E. and Schulten, K. (2001) Structure 9, 1083-1093.

[67] Jensen, M.Ø., Park, S., Tajkhorshid, E. and Schulten, K. (2002) Proc. Natl. Acad. Sci. USA 99, 6731-6736.

[68] Fujiyoshi, Y., Mitsuoka, K., de Groot, B.L., Philippsen, A., Grubmüller, H., Agre, P. and Engel, A. (2002) Curr. Opin. Struct. Biol. 12, 509-515.

[69] Viani, M.B., Schäfer, T.E., Chand, A., Rief, M., Gaub, H. and Hansma, P.K. (1999) J. Appl. Phys. 86, 2258-2262.

[70] Walz, T., Smith, B., Zeidel, M., Engel, A. and Agre, P. (1994) J. Biol. Chem. 269, 1583-1586.

[71] Walz, T.Proc.Na (1997) Nature 387, 624-627.

[72] Mitsuoka, K., Murata, K., Walz, T., Hirai, T., Agre, P., Heymann, J.B., Engel, A. and Fujiyoshi, Y. (1999) J. Struct. Biol. $128,34-43$. 